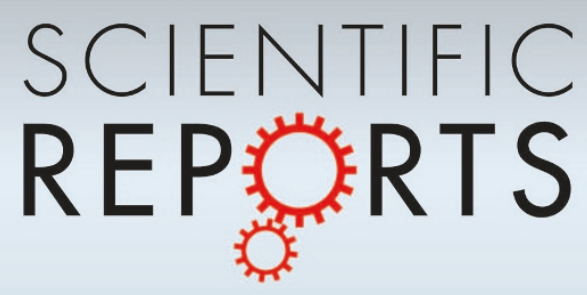

\section{OPEN}

SUBJECT AREAS:

ECOLOGICAL GENETICS

EXPERIMENTAL EVOLUTION

Received

20 November 2013

Accepted

24 February 2014

Published

18 March 2014

Correspondence and requests for materials should be addressed to D.C. (chinachudong@ sina.com) or Y.J.Z. (zhangyoujun@caas. $\mathrm{cn})$

\title{
Evidence For Rapid Spatiotemporal Changes in Genetic Structure of an Alien Whitefly During Initial Invasion
}

\author{
Dong Chu', Dong Guo', Yunli Tao', Defeng Jiang' ' Jie Li' \& Youjun Zhang ${ }^{2}$
}

\begin{abstract}
${ }^{1}$ Key Lab of Integrated Crop Pest Management of Shandong Province, College of Agronomy and Plant Protection, Qingdao Agricultural University, Qingdao, 266109, P. R. China, ${ }^{2}$ Department of Plant Protection, Institute of Vegetables and Flowers, Chinese Academy of Agricultural Sciences, Beiijing, 100081, P. R. China.
\end{abstract}

The sweetpotato whitefly Bemisia tabaci $Q$ species is a recent invader and important pest of agricultural crops in China. This research tested the hypothesis that the $Q$ populations that establish in agricultural fields in northern China each year are derived from multiple secondary introductions and/or local populations that overwinter in greenhouses (the pest cannot survive winters in the field in northern China). Here, we report the evidence that the $Q$ populations in agricultural fields mainly derive from multiple secondary introductions. In addition, the common use of greenhouses during the winter in certain locations in northern China helps increase the genetic diversity and the genetic structure of the pest. The genetic structure information generated from this long-term and large-scale field analysis increases our understanding of $B$. tabaci $Q$ as an invasive pest and has important implications for B. tabaci $Q$ management.

$\mathrm{V}$ arious factors, such as invasion history, pest dispersal ability, founder numbers, and passive dispersal by human activity ${ }^{1-4}$, affect the genetic structure and diversity of invasive pests. Introduced populations often experience rapid genetic differentiation and microevolution as they adapt to the new environmental conditions $s^{4,5}$. Knowledge of the population genetics can be used for predicting invasiveness and the efficacy of alternative control efforts and improving management of invasive species 5 . Although a substantial body of research exists describing the structure and diversity of an alien species after establishment, data regarding diversification during initial invasion are limited. An important reason for this is that the introduction often remains unrecognised until the alien population becomes very large 6 . Population genetic structure and diversity can also be readily affected by spatial and temporal factors ${ }^{7-11}$, and several studies have highlighted the need for long-term and large-scale analyses ${ }^{12-14}$.

The sweetpotato whitefly, Bemisia tabaci (Gennadius), is an important agricultural pest worldwide and is regarded as a species complex comprising at least 28 cryptic species ${ }^{15-17}$. Some of these cryptic species have become invasive where they have been introduced. One species complex member, designated MED and commonly known as B. tabaci biotype Q (hereafter referred to as $B$. tabaci $\mathrm{Q}$ or $\mathrm{Q}$ ), has been introduced to many countries from its original Mediterranean distribution, including China, over the past decade ${ }^{16}$. However, the various introduced B. tabaci $\mathrm{Q}$ populations have differed in invasive ability ${ }^{18-21}$ and other biological traits such as those related to virus transmission ${ }^{22,23}$. Genetic analysis of the introduced populations is a promising strategy to discern the microevolutionary and ecological adaptations that underlie the ability of this insect pest to invade agro-ecosystems. Such knowledge is important for the design and optimisation of sustainable pest management strategies $^{1-4}$.

In China, B. tabaci Q was first detected in Yunnan, Beijing, and Henan provinces in $2003^{18}$. During subsequent years, $B$. tabaci $\mathrm{Q}$ has gradually displaced the previously well-established populations of MEAM1 (commonly known as B. tabaci biotype B) and has been the dominant species in most regions of China since $2008^{19,20}$. Since 2005, the invasion process of $B$. tabaci Q has been well documented in many locations in Shandong Province in northern China ${ }^{24-26}$, and since this initial detection, we have continuously sampled whitefly populations from numerous Shandong locations ${ }^{24}$. As is the case for other areas of China, the percentage of $\mathrm{Q}$ in $B$. tabaci populations has gradually increased, and $\mathrm{Q}$ has been the dominant species in most locations in Shandong Province since $2008^{25,26}$. B. tabaci $\mathrm{Q}$ cannot survive the winter in the field in Shandong ${ }^{27}$, but can survive in greenhouses, which are commonly used in the area of Shouguang. Despite elimination by low 


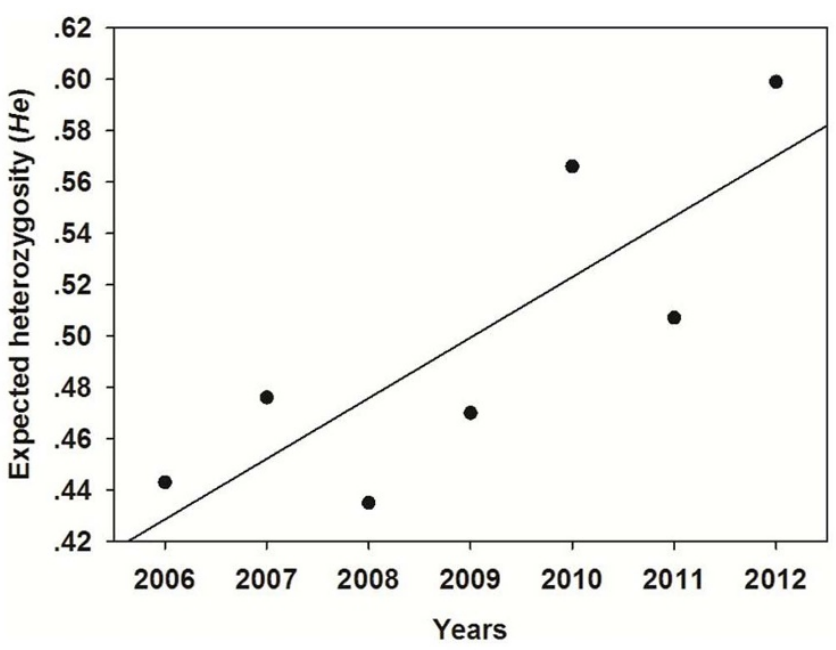

Figure $1 \mid$ Temporal changes in genetic diversity $(\mathrm{He})$ in the populations of B. tabaci Q in Shouguang, Shandong Province during 2006-2012.

winter temperatures, Q has been present and abundant in the field during every growing season since its initial appearance in the province.

To explain how Q can appear in the field year after year in spite of its inability to overwinter in the field, we hypothesise that the initial populations detected in the field (i.e., from 2006 to 2012) derived from multiple, secondary introductions and/or from populations that overwintered in local greenhouses in Shouguang. The purpose of the present study is to test this hypothesis.

\section{Results}

Changes in genetic diversity and in microsatellite allele frequencies. From 2006 to 2012 in Shandong Province, the genetic diversity of $B$. tabaci Q (i.e. the value of $\mathrm{He}$ ) increased over time only in one location (Shouguang) (Fig. 1) $\left(R^{2}=0.673, P=0.024\right)$, which is the location where greenhouses are widely used during winter. However, the values of $\mathrm{He}$ in the six other locations were not significantly correlated with time $(P>0.05)$. In addition, the relationship between average $H e$ and time was not statistically significant $\left(R^{2}=0.486, P=0.082\right)$ (Fig. S1).

Analysis of genetic structure. Of the 45 collections, 44 exhibited significant departures from Hardy-Weinberg equilibrium and had high and positive mean values of Fis (0.287-0.552), indicating the existence of heterozygote deficiencies (Table 1). With respect to pairwise comparisons between collections, 841 of 990 values of Fst $(84.8 \%)$ were associated with a significant exact test (Table S1).

The results of a hierarchical analysis of molecular variance (AMOVA) revealed significant differences according to sample time (Table 2) and indicated no significant genetic difference between locations (Table 2). Further investigation showed a significant difference in the genetic structure of $B$. tabaci $Q$ as time progressed from 2006 to 2012. Of the 21 values of FCT (difference among groups), only seven were not significant (Table S2).

Analyses using BAPS software identified ten genetic clusters within the 45 collections (Figs. 2 and S2). In each year, 2-4 genetic clusters were observed with samples from the different locations. In 2006, three distinct genetic clusters were present for all four collections that were sampled in that year, but in 2007, the 2006 clustering pattern was replaced by new cluster formations for all four locations. In 2008, the 2007 clusters were replaced by novel clustering patterns in three of six locations, and in 2009, the genetic clusters from 2008 persisted in all locations except Linyi. In 2010, the 2009 clusters were replaced by other clusters at all locations, and in 2011, the 2010 clusters had, once again, been replaced by other clusters at all locations. In 2012, the genetic clusters from 2011 had been replaced by other clusters in four of the seven locations.

\section{Discussion}

The specimens we collected during 2006-2012 provide an excellent opportunity to examine the changes in $B$. tabaci Q genetic diversity and structure. Because B. tabaci Q cannot overwinter in the field in northern China, we hypothesised that the initial populations detected in the field each year derived from multiple, secondary introductions and/or from populations that overwintered in local greenhouses. To test this hypothesis, we analysed the spatiotemporal genetic changes of field populations using 7 years of microsatellite data from samples collected annually at seven locations in Shandong Province, China.

Rapid spatiotemporal changes in genetic diversity. The microsatellite DNA data describing genetic diversity and allele frequency presented here reveal a significant genetic shift at the seven field locations during the initial invasion of the whitefly B. tabaci Q in Shandong Province, China. At most locations, the genetic diversity $\mathrm{He}$ ) remained constant from 2006 to 2009, increased substantially from 2010 to 2011, and decreased slightly in 2012 (Table 1). The values of microsatellite allele frequencies and average number of alleles per locus $(\mathrm{Na})$ were consistent with the trends in $\mathrm{He}$.

The changes in genetic variation in $B$. tabaci $\mathrm{Q}$ collections were unexpected. We expected that shortly after populations became established, those in different locations in Shandong Province would experience selection due to insecticide exposure and wintering in the greenhouse, and that these selective forces would reduce genetic diversity. In support of this expectation, Chu et al. $(2008)^{28}$ found that selection by the insecticide thiamethoxam decreased the genetic diversity of $B$. tabaci under laboratory conditions. Franklin et al. $(2010)^{10}$ reported that heterozygosity levels in greenhouse populations of Trichoplusia ni were slightly lower in the spring than in the summer and fall, perhaps as a result of winter cleanup operations.

The increases in the genetic diversity indices may be associated with the repeated yearly introduction of alien whiteflies from other regions as a consequence of natural dispersal or human activities. Our results also suggest that the number of alien whiteflies introduced was highly variable over time, i.e., the number introduced was small during 2006-2009 and in 2012, but was large during 20102011. An increase in genetic diversity caused by new introductions of alien whiteflies, however, could be countered by winter clean up in greenhouses.

An interesting finding in this study was that the location with abundant greenhouses was the only location in which genetic diversity was significantly correlated with time (expressed as years). We infer that populations overwintering in greenhouses may play an important role in increasing the genetic diversity of $B$. tabaci $\mathrm{Q}$ in Shouguang, northern China.

Rapid spatiotemporal changes in genetic structure. The Fst values reveal substantial genetic structure in the collections of $B$. tabaci $Q$ at the seven locations. These results indicate that gene flow was low between locations and that inbreeding was prevalent at most locations, which is consistent with Tsagkarakou et al. $(2007)^{29}$, who documented low gene flow between $B$. tabaci $\mathrm{Q}$ populations in Greece that were separated by only a few kilometres. These data suggest that gene flow between whitefly populations may be minimal and that dispersal by B. tabaci Q is usually limited. Low gene flow between populations may contribute to the genetic differentiation of the populations across the region. BAPS analysis revealed the existence of at least ten genetic clusters of $B$. tabaci $\mathrm{Q}$ in Shandong Province.

The most interesting result in our study was the rapid change in population genetic structure, which was significantly associated with 
00000000000000000000000000000000000000000000

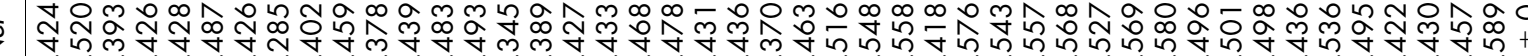
0000000000000000000000000000000000000000000000

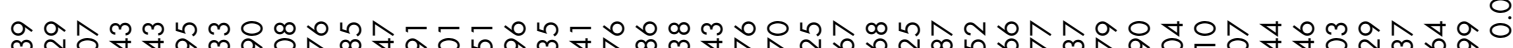

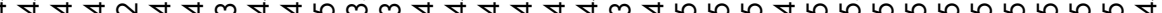

0000000000000000000000000000000000000000000000

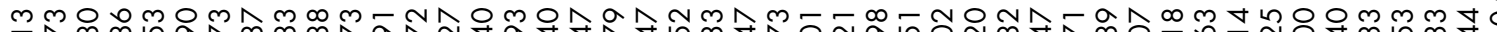

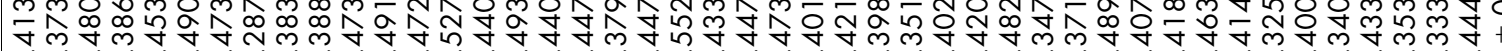
$000000000000000000000000000000000000000000000 \%$

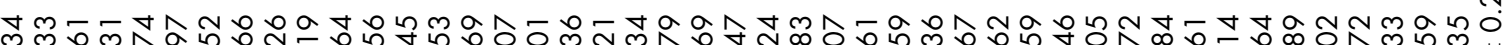
-NOAN

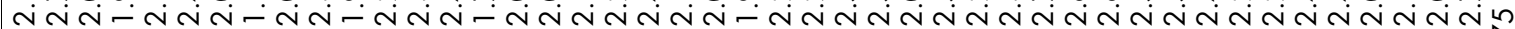

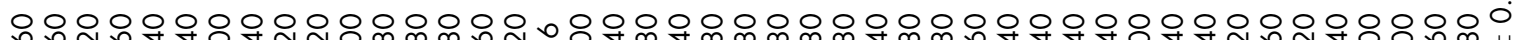

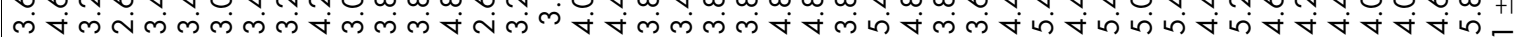
(1)

$$
\text { . }
$$

$$
\text { N }
$$


Table 2 | Hierarchical AMOVA table and corresponding values for FCT (difference among groups), FSC (differences among collections within groups), and FST (differences among all collections)

\begin{tabular}{lccc} 
Hierarchical structure & FCT & FSC & FST \\
\hline Location $>$ Time & -0.00398 & $0.17006 * *$ & $0.16676 * *$ \\
Time $>$ Location & $0.08570 * *$ & $0.09948 * *$ & $0.17666 * *$ \\
\hline$* P \leq 0.05, * * P \leq 0.001$. & & &
\end{tabular}

time rather than location according to hierarchical AMOVA (Table 2). According to BAPS analysis for the data from 2006 to 2012, specific genetic clusters persisted for only $1-3$ years in any one location (Fig. 2), which suggests that genetic composition changed rapidly at those locations. This result is consistent with the genetic changes in B. tabaci B over a short time frame in the Lockyer Valley, Queensland, Australia ${ }^{11}$. In that study, Dinsdale and colleagues used eight microsatellite loci and detected a significant temporal change in the local genetic composition of B. tabaci B during the growing seasons of 2006-2007.

The mechanism underlying the rapid spatiotemporal changes in genetic structure of B. tabaci $\mathrm{Q}$ in Shandong Province is consistent with the hypothesis that the populations detected in the field from 2006 to 2012 were derived from multiple, secondary introductions and from populations that overwintered in local greenhouses. In the present study, host and climate factors affecting the genetic composition were similar across the study area because the specimens were collected from a limited number of host types and within the same region; thus it seems unlikely that host or climate can explain the changes and differences in genetic structure of B. tabaci $\mathrm{Q}$. At the Liaocheng location, for example, the host plants were eggplant and cotton throughout the sampling period (2007-2012) but the genetic structure changed. In support of the inference that there were multiple introductions of the pest, some new genetic clusters suddenly appeared in different locations in the same year, e.g., three new genetic clusters appeared in 2010 or 2011 . Local whitefly populations that overwinter in greenhouses may also affect the genetic structure, e.g., the population in Shouguang in 2012 (12-SG), whose genetic cluster differed from those of the other six locations in 2012, may be a mixture of the local population and external whitefly individuals. As a typical location with greenhouses where whiteflies can survive the winter, Shouguang may have numerous local whitefly individuals that survive the winter.

The application of insecticides cannot be ruled out as a factor influencing genetic change because insecticides are widely used in the study region. Prior studies suggest that the application of some insecticides can cause population bottlenecks that enhance genetic differentiation ${ }^{10,28,30}$.

Implications for B. tabaci $\mathrm{Q}$ management and for future research. The finding of rapid genetic changes in field populations of $B$. tabaci $Q$ during the initial invasion provides useful information concerning the insect's invasion mechanism and its control. First, the results indicate that both secondary introductions from other regions and overwintering individuals from local greenhouses can contribute to the substantial genetic variability, which would provide a genetic basis for the adaptation of $B$. tabaci $\mathrm{Q}$ to new environments. Second, a change in genetic cluster may be associated with a change in biological and ecological characteristics. Understanding the relationship between the genotypes and these characteristics, and the monitoring of genotypes, can help guide management of $B$. tabaci $^{18-23}$. For example, if a new genetic cluster involves a high level of resistance to specific insecticides, the types of insecticides can be adjusted accordingly.

Several questions remain regarding the genetic changes of invasive B. tabaci $\mathrm{Q}$ in China. First, we do not know the geographic distribution of the rapid genetic changes of $B$. tabaci $Q$ populations; additional research is required to determine whether the changes in the genetic clusters in Shandong Province reflect the changes at the

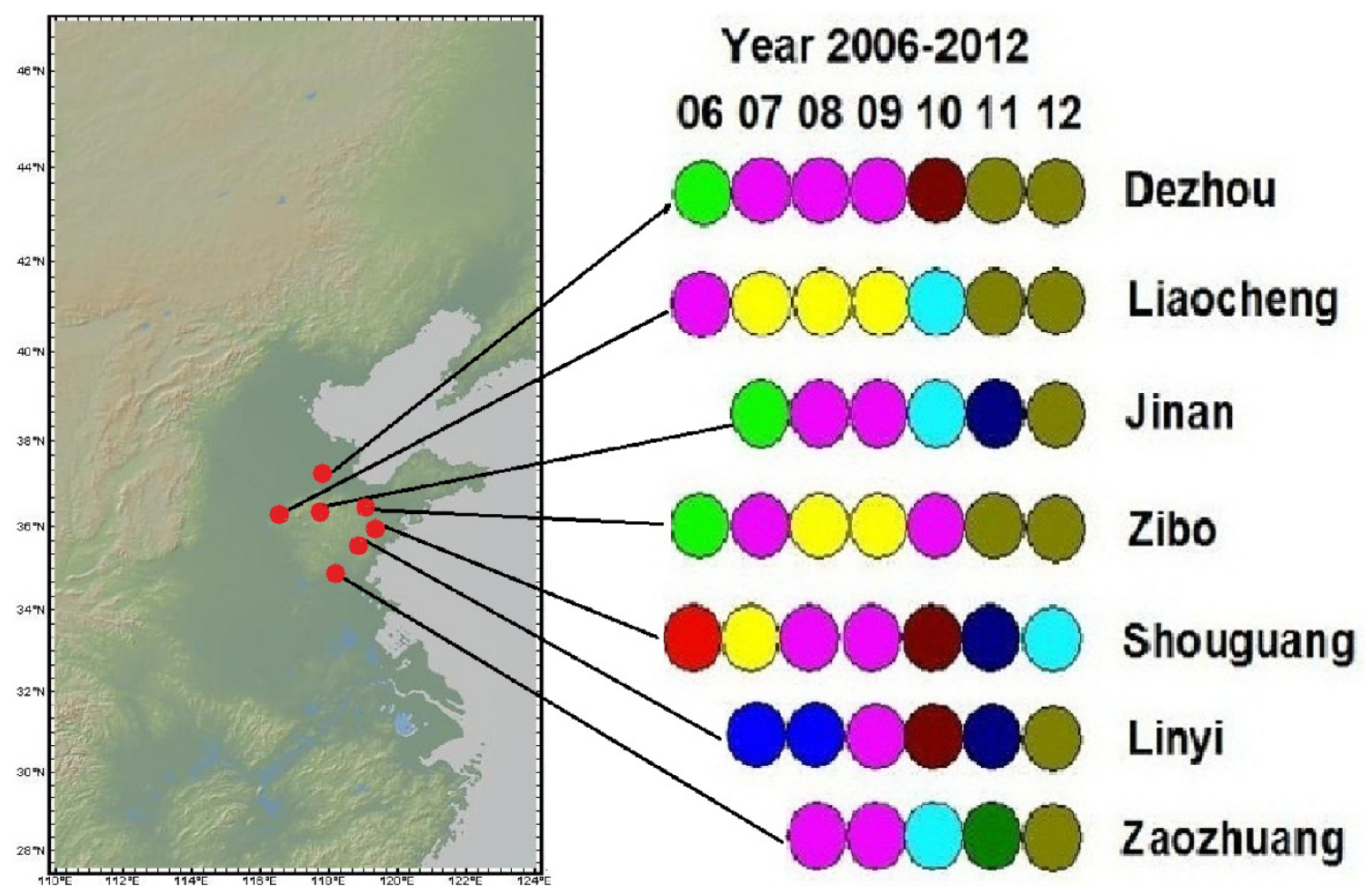

Figure $2 \mid$ B. tabaci Q sampling locations in Shandong Province during 2006-2012. The color-coded assignment of populations to ten clusters identified by BAPS is shown in Figure S2 (B). In the graph, each color represents one cluster. The sampling locations are grouped by color to indicate which groups are likely to represent distinct populations. The map was generated using GeoMapApp (version 2) (http://www.geomapapp.org/). 
national scale. Second, the key factors affecting the spatiotemporal genetic changes remain to be determined, although many ecological factors have been explored or discussed for other species ${ }^{10,11,30}$. Finally, the potential effects of population genetic changes on the biological characteristics of the populations should be explored. Two important characteristics concern the harbouring of endosymbionts and the transmission of plant viruses. The presence of the endosymbiont Rickettsia in whiteflies enhances whitefly fitness and spread $^{31}$, and variation in the percentage of invasive $B$. tabaci $\mathrm{Q}$ with secondary endosymbionts was associated with temporal genetic changes in B. tabaci $\mathrm{Q}$ in China ${ }^{4}$. Similarly, the acquisition of certain plant viruses by whiteflies can modify whitefly feeding behaviour ${ }^{32}$.

\section{Methods}

Sample collection. During July to September of 2006-2012, adult B. tabaci were collected from host plants including four field crops (cotton, tomato, eggplant, and cucumber) and one weed (Japanese hop) at seven representative locations (Dezhou, DZ; Liaocheng, LC; Jinan, JN; Shouguang, SG; Zibo, ZB; Zaozhuang, ZZ; and Linyi, LY) in Shandong Province, China (Table 1). The geographical locations are shown using GeoMapApp v. $2^{33}$ (Fig. 2). Among the seven locations, Shouguang is considered China's main vegetable producer, and the abundant greenhouses in Shouguang are used to produce vegetables throughout the winter (http://en. wikipedia.org/wiki/Shouguang). Each B. tabaci collection involved sampling whiteflies from every second available plant until at least 100 whitefly adults had been collected from each host. The living B. tabaci individuals were stored in $95 \%$ ethanol at $-20^{\circ} \mathrm{C}$ until DNA extraction. Four collections were missing (Jinan and Linyi were not sampled in 2006, and Zibo was not sampled in 2006 or 2007), and there were, therefore, 45 rather than 49 collections in total.

DNA extraction, PCR amplification, and species determination. Genomic DNA was extracted from individual females (14-30 individuals) from each collection (one collection was defined as the samples from a single location in a single year), as described by Frohlich et al. (1999) ${ }^{34}$. The extracted genomic DNA was stored at $-20^{\circ} \mathrm{C}$ and used as template for PCR amplification. Our long-term field survey indicated that only two species from the B. tabaci species complex (B. tabaci B and Q) were represented in Shandong Province ${ }^{35,36}$. B. tabaci species was determined using cleaved amplified polymorphic sequences (CAPS) of the mitochondrial cytochrome oxidase I (mtCOI) gene (about $620 \mathrm{bp}$ ), which was amplified with primers (C1-J2195/R-BQ-2819), as reported by Chu et al. $(2011)^{26}$. The amplified mtCOI fragment was cleaved by the restriction endonuclease $V s p \mathrm{I}^{37} ; \mathrm{mtCOI}$ fragments that can be cut by VspI belong to B. tabaci $\mathrm{Q}$ and those that cannot be cut belong to B. tabaci $\mathrm{B}^{26}$.

Microsatellite genotyping. Microsatellite markers have been widely used to analyse the population structure of $B$. $t a b a c i^{11,26,38,39}$. In this study, primers for a suite of five microsatellites (BEM6, BEM11, BEM25, BEM31, and BEM37) were used to amplify loci from 1,251 B. tabaci Q individuals collected in Shandong Province during 20062012 , as described by De Barro et al. $(2003)^{40}$. Amplification products of the microsatellite loci were run on an ABI 3730xl DNA analyser. Allele size was determined by comparing the mobility of the PCR products to that of the GeneScanTM 400HD size standard (Applied Biosystems).

Data analysis. For each of the 45 microsatellite data sets (seven locations) of B. tabaci $\mathrm{Q}$, the following genetic diversity indexes were calculated using POPGENE v.1.31 software $^{41}$ : observed number of alleles $(\mathrm{Na})$, effective number of alleles $(\mathrm{Ne})$, expected heterozygosity $(\mathrm{He})$, observed heterozygosity $(\mathrm{Ho})$, and Nei's expected heterozygosity $(\mathrm{Nei})$. The fixation index within collection (Fis) was calculated to quantify the inbreeding coefficient within each collection with GENEPOP v.3.4 $4^{42}$. Conformity to Hardy-Weinberg equilibrium was assessed with exact tests in GENEPOP v.3.4 with Markov chain parameters of 10,000 dememorisation steps, followed by 1,000 batches of 10,000 iterations per batch. Deviations from heterozygosity deficits were assessed using GENEPOP v. $3.4^{42}$.

The fixation index between collections ( Fst $)$ was estimated with GENEPOP v.3.4. $4^{42}$. A hierarchical AMOVA was performed with ARLEQUIN v.3.5 software to compare the values for FCT (difference among groups), FSC (difference among collections within groups), and FST (differences among all collections) ${ }^{43}$. In this analysis, 'groups' refers to data grouped according to location or time. AMOVA computations were performed with 10,000 permutations to test for significance.

Genetic clusters within the $45 \mathrm{~B}$. tabaci Q collections and estimated individual coefficients of ancestry were determined using BAPS v.4.14 software ${ }^{44}$. For estimating individual ancestry coefficients via admixture analyses, the recommended values were used as follows: the number of iterations used to estimate the admixture coefficients for the individuals was 100; the number of reference individuals from each collection was 200; and the number of iterations used to estimate the admixture coefficients for the reference individuals was 20 .

1. Timm, A. E., Geertsema, H. \& Warnich, L. Population genetic structure of Grapholita molesta (Lepidoptera: Tortricidae) in South Africa. Ann. Entomol. Soc. Amer. 101, 197-203 (2008).
2. Chen, M. H. \& Dorn, S. Microsatellites reveal genetic differentiation among populations in an insect species with high genetic variability in dispersal, the codling moth, Cydia pomonella (L.) (Lepidoptera: Tortricidae). Bull. Entomol. Res. 100, 75-85 (2010)

3. Torriani, M. V. G., Mazzi, D., Hein, S. \& Dorn, S. Structured populations of the oriental fruit moth in an agricultural ecosystem. Mol. Ecol. 19, 2651-2660 (2010).

4. Kirk, H., Dorn, S. \& Mazzi, D. Molecular genetics and genomics generate new insights into invertebrate pest invasions. Evol. Appl. 6, 842-856 (2013)

5. Sakai, A. K. et al. The population biology of invasive species. Ann. Rev. Ecol. Syst. 32, 305-332 (2001)

6. Lockwood, J. L., Hoopes, M. F. \& Marchetti, M. P. Invasion Ecology. Blackwell Press, Malden, MA (2007).

7. De Barro, P. J. et al. Spatial and temporal genetic variation in British field populations of the grain aphid Sitobion avenae (F) (Hemiptera: Aphididae) studied using RAPD-PCR. P. Roy. Soc. B-Biol. Sci. 262, 321-327 (1995).

8. Vorburger, C. Temporal dynamics of genotypic diversity reveal strong clonal selection in the aphid Myzus persicae. J. Evolution. Biol. 19, 97-107 (2006).

9. Münzbergová, Z. et al. Spatial and temporal variation in dispersal pattern of an invasive pine. Biol. Invasions 12, 2471-2486 (2010).

10. Franklin, M. T., Ritland, C. E. \& Myers, J. H. Spatial and temporal changes in genetic structure of greenhouse and field populations of cabbage looper, Trichoplusia ni. Mol. Ecol. 19, 1122-1133 (2010).

11. Dinsdale, A. et al. Rapid genetic turnover in populations of the insect pest Bemisia tabaci Middle East: Asia Minor 1 in an agricultural landscape. Bull. Entomol. Res. 102, 539-549 (2012)

12. Scott, K. D. et al. Assessing moth migration and population structuring in Helicoverpa armigera (Lepidoptera: Noctuidae) at the regional scale: example from the Darling Downs, Australia. J. Econ. Entomol. 98, 2210-2219 (2005).

13. Scott, K. D. et al. Gene-flow between populations of cotton bollworm Helicoverpa armigera (Lepidoptera: Noctuidae) is highly variable between years. Bull. Entomol. Res. 95, 381-392 (2005)

14. Carrière, Y. et al. Large-scale, spatially-explicit test of the refuge strategy for delaying insecticide resistance. Pro. Natl. Acad. Sci. USA. 109, 775-780 (2012).

15. Dinsdale, A. et al. Refined global analysis of Bemisia tabaci (Hemiptera: Sternorrhyncha: Aleyrodoidea: Aleyrodidae) mitochondrial cytochrome oxidase 1 to identify species level genetic boundaries. Ann. Entomol. Soc. Am. 103, 196-208 (2010)

16. De Barro, P. J., Liu, S. S., Boykin, L. M. \& Dinsdale, A. B. Bemisia tabaci: a statement of species status. Ann. Rev. Entomol. 56, 1-19 (2011).

17. Hu, J. et al. An extensive field survey combined with a phylogenetic analysis reveals rapid and widespread invasion of two alien whiteflies in China. PLOS ONE 6, e16061 (2011).

18. Chu, D. et al. The introduction of the exotic Q biotype of Bemisia tabaci (Gennadius) from the Mediterranean region into China on ornamental crops. Fla. Entomol. 89, 168-174 (2006).

19. Teng, X., Wan, F. H. \& Chu, D. Bemisia tabaci biotype Q dominates other biotypes across China. Fla. Entomol. 93, 363-368 (2010).

20. Pan, H. P. et al. Further spread of and domination by Bemisia tabaci biotype Q on field crops in China. J. Econ. Entomol. 104, 978-985 (2011).

21. Dickey, A. M. et al. Population genetics of invasive Bemisia tabaci (Hemiptera: Aleyrodidae) cryptic species in the United States based on microsatellite markers. J. Econ. Entomol. 106, 1355-1364 (2013).

22. Gottlieb, Y. et al. The transmission efficiency of tomato yellow leaf curl virus by the whitefly Bemisia tabaci is correlated with the presence of a specific symbiotic bacterium species. J. Virol. 84, 9310-9317 (2010).

23. Pan, H. P. et al. Differential effects of an exotic plant virus on its two closely related vectors. Sci. Rep. 3, 2230 (2013).

24. Chu, D. et al. Biotype status and distribution of Bemisia tabaci (Hemiptera: Aleyrodidae) in Shandong province of China based on mitochondrial DNA markers. Env. Entomol. 36, 1290-1295 (2007).

25. Chu, D. et al. Further insights into the strange role of bacterial endosymbionts in whitefly, Bemisia tabaci: Comparison of secondary symbionts from biotypes B and Q in China. Bull. Entomol. Res. 101, 477-486 (2011).

26. Chu, D. et al. Investigation of the genetic diversity of an invasive whitefly in China using both mitochondrial and nuclear DNA markers. Bull. Entomol. Res. 101, 467-475 (2011).

27. Ren, S. X. et al. Research progress of the monitoring, forecast and sustainable management of whitefly pests in China. Chinese J. Appl. Entomol. 48, 7-15 (2011)

28. Chu, D. et al. Thiamethoxa application can decrease the genetic diversity of Bemisia tabaci (Gennadius) biotype B. Acta Entomologica Sinica 51, 150-154 (2008).

29. Tsagkarakou, A. et al. Biotype status and genetic polymorphism of the whitefly Bemisia tabaci (Hemiptera: Aleyrodidae) in Greece: mitochondrial DNA and microsatellites. Bull. Entomol. Res. 97, 29-40 (2007).

30. Franck, P., Reyes, P. F. M., Olivares, J. \& Sauphanor, B. Genetic architecture in codling moth populations: comparison between microsatellite and insecticide resistance markers. Mol. Ecol. 16, 3554-3564 (2007).

31. Himler, A. G. et al. Rapid spread of a bacterial symbiont in an invasive whitefly is driven by fitness benefits and female bias. Science 332, 254-256 (2011).

32. Liu, B. M. et al. Multiple forms of vector manipulation by a plant-infecting virus: Bemisia tabaci and tomato yellow leaf curl virus. J. Virol. 87, 4929-4937 (2013). 
33. Ryan, W. et al. Global Multi-Resolution Topography Synthesis. Geochem. Geophys. Geosyst. 10, (Q03014) (2009).

34. Frohlich, D. R. et al. A phylogeographical analysis of the Bemisia tabaci species complex based on mitochondrial DNA markers. Mol. Ecol. 8, 1683-1691 (1999).

35. Chu, D., Zhang, Y. J. \& Wan, F. H. Cryptic invasion of the exotic Bemisia tabaci biotype Q occurred widespread in Shandong Province of China. Fla. Entomol. 93, 203-207 (2010).

36. Chu, D., Wan, F. H., Zhang, Y. J. \& Brown, J. K. Change in the biotype composition of Bemisia tabaci in Shandong Province of China from 2005 to 2008. Env. Entomol. 39, 1028-1036 (2010).

37. Khasdan, V.et al. DNA markers for identifying biotypes B and Q of Bemisia tabaci (Hemiptera: Aleyrodidae) and studying population dynamics. Bull. Entomol. Res. 95, 605-613 (2005)

38. De Barro, P. J. Genetic structure of the whitefly Bemisia tabaci in the Asia-Pacific region revealed using microsatellite markers. Mol. Ecol. 14, 3695-3718 (2005).

39. Delatte, H. et al. Microsatellites reveal extensive geographical, ecological and genetic contacts between invasive and indigenous whitefly biotypes in an insular environment. Genet. Res. 87, 109-124 (2006).

40. De Barro, P. J. et al. Isolation and characterization of microsatellite loci in Bemisia tabaci. Mol. Ecol. Notes 3, 40-43 (2003).

41. Yeh, F. C. et al. POPGENE, the user-friendly shareware for population genetic analysis. Molecular Biology and Biotechnology Centre, University of Alberta, Edmonton, Canada (1997)

42. Raymond, M. \& Rousset, F. GENEPOP (version 1.2): population genetics software for exact tests and ecumenicism. J. Hered. 86, 248-249 (1995).

43. Excoffier, L., Laval, G. \& Schneider, S. Arlequin (version 3.0): an integrated software package for population genetics data analysis. Evol. Bioinform. Online 1, 47-50 (2005).

44. Corander, J., Siren, J. \& Arjas, E. Bayesian spatial modeling of genetic population structure. Comput. Stat. 23, 111-129 (2008).

\section{Acknowledgments}

We thank A. Dinsdale (The University of Queensland, School of Biological Sciences, Australia) and M. H. Chen (Northwest A\&F University, China) for help in the revision of this manuscript. This research was supported by the High-Level Talents Fund of Qingdao Agricultural University (631212), the National Natural Science Foundation of China (31272105), the Science and Technology Development Planning Program of Qingdao (13-1-3-108-nsh), the Taishan Mountain Scholar Constructive Engineering Foundation of Shandong to D. Chu, and the National Science Fund for Distinguished Young Scholars of China (31025020) to Y. Zhang. The funders had no role in study design, data collection and analysis, decision to publish, or preparation of the manuscript.

\section{Author contributions}

D.C. and Y.J.Z. designed the experiment. D.C. and D.G. performed the experiment. Y.L.T., J.L. and D.F.J. contributed reagents/materials. D.C. and Y.J.Z. wrote the paper.

\section{Additional information}

Supplementary information accompanies this paper at http://www.nature.com/ scientificreports

Competing financial interests: The authors declare no competing financial interests.

How to cite this article: Chu, D. et al. Evidence For Rapid Spatiotemporal Changes in Genetic Structure of an Alien Whitefly During Initial Invasion. Sci. Rep. 4, 4396; DOI:10.1038/srep04396 (2014)

(c) (i) $(-)$ This work is licensed under a Creative Commons Attributionvisit http://creativecommons.org/licenses/by-nc-nd/3.0 